\title{
Mapping the Milky Way structure with methanol and water masers
}

Luca Moscadelli*

INAF - Osservatorio Astrofisico di Arcetri

E-mail: mosca@arcetri.astro.it

\section{Mark J. Reid}

Harvard-Smithsonian Center for Astrophysics

E-mail: reidecfa. harvard.edu

\section{Karl M. Menten}

Max-Planck-Institut für Radioastronomie

E-mail: kmenten@mpifr-bonn.mpg.de

\section{Andreas Brunthaler}

Max-Planck-Institut für Radioastronomie

E-mail: brunthal@mpifr-bonn.mpg.de

\section{Xing Wu Zheng}

Department of Astronomy, Nanjing University

E-mail: xwzheng@mail.nju.edu.cn

\section{Ye Xu}

Purple Mountain Observatory, Chinese Academy of Science

E-mail: xuye@pmo.ac.cn

\begin{abstract}
We review recent VLBA and VERA results in measuring trigonometric parallaxes of methanol and water maser sources. The derived source distances are typically accurate to 5-10\%, and in a few cases even better. Proper motions are measured with typical uncertainties of a few kilometer per second. The VLBA data observed so far allow one to precisely locate portions of the Perseus Arm, the Local Spur and Carina-Sagittarius Arm. VERA and VLBA observations support the presence of an Outer Arm extending from the $2^{\text {nd }}$ to the $3^{\text {rd }}$ quadrant of the Milky Way. Derived peculiar motions suggest that the observed maser sources orbit slower than the Galaxy spins.
\end{abstract}

The 9th European VLBI Network Symposium on The role of VLBI in the Golden Age for Radio Astronomy and EVN Users Meeting

September 23-26, 2008

Bologna, Italy

\footnotetext{
* Speaker.
} 


\section{Introduction}

Imaging the Milky Way, an observer in another galaxy would probably note a spiral structure dotted with many bright HII regions. Presently it is believed that the Milky Way is a spiral galaxy, and a best "educated guess" is that it is a barred Sb to Sc galaxy (Blitz, Fich \& Kulkarni 1983; Gerhard 2002). Since we are inside the Milky Way, the task of properly characterizing its structure has proved very difficult (Bash 1981).

Originally, HI emission was used to map the structure of the Milky Way (Oort, Kerr \& Westerhout 1958). Longitude-velocity plots of HI emission clearly demonstrated that there were some coherent, large-scale structures, which were interpreted in terms of spiral arms in the Milky Way. However, the difficulty of determining accurate distances to HI clouds made the task of turning longitude-velocity data into a true plan-view of the Milky Way very uncertain (Burton 1988). Later, millimeter-wave observations of molecules, such as $\mathrm{CO}$, also evidenced coherent, largescale structures with higher contrast than seen in HI (Dame, T. M., Hartmann, D. \&Thaddeus, P. 2001). But, again, the uncertainty of distances to molecular clouds was the main obstacle to map the Milky Way with sufficient accuracy to reveal its spiral structure.

Georgelin \& Georgelin (1976), hereafter GG76, combined optical observations of young stars and radio data of HI cloud and HII region emissions, to produce a "plan-view" model of the spiral structure of the Milky Way. Luminosity distances to nearby stars were used where available and kinematic distances elsewhere, mostly for more distant HII regions. While subject to very significant uncertainties from kinematic distances, the GG76 model has remained the basis for the "standard" model of the spiral structure of the Milky Way for nearly 30 years. More recently, Taylor \& Cordes (1993) have modeled pulsar dispersion measures to refine the GG76 model (see Fig. 1).

\section{Maser Trigonometric Parallaxes}

Methanol $\left(\mathrm{CH}_{3} \mathrm{OH}\right)$ and water $\left(\mathrm{H}_{2} \mathrm{O}\right)$ masers are excellent astrometric targets for parallax measurements. Both maser types are widespread and associated with newly formed stars in regions of high-mass star formation. The class $\mathrm{II} \mathrm{CH}_{3} \mathrm{OH}$ masers (e.g. 6.7 and $12 \mathrm{GHz}$ transitions) generally are compact ( $\sim 1$ mas), slow moving, and vary slowly, which minimizes the possibility of brightness variations mimicing position shifts. $\mathrm{H}_{2} \mathrm{O}$ masers are also compact and strong, and the high frequency of the transition minimizes ionospheric fluctuations and interstellar scattering problems. However, $\mathrm{H}_{2} \mathrm{O}$ masers can be highly variable on time scales as short as weeks to months and, since parallax observations are best made over a timespan of $1 \mathrm{yr}$, this can be problematic.

VLBI (Very Long Baseline Interferometry) observations can presently achieve the accuracy to measure the trigonometric parallax of masers through the whole galactic disk. Notably, the NRAO ${ }^{1}$ Very Long Baseline Array (VLBA) and the Japanese project VLBI Exploration of Radio Astrometry (VERA) (Kobayashi et al. 2005) have already demonstrated the ability of measuring relative positions with an accuracy of $\sim 10 \mu$ as (Reid et al. 2008; Honma et al. 2007), which allows one to measure parallaxes to distances up to $10 \mathrm{kpc}$. A noteable result that demonstates the reliability

\footnotetext{
${ }^{1}$ The National Radio Astronomy Observatory is a facility of the National Science Foundation operated under cooperative agreement by Associated Universities, Inc.
} 


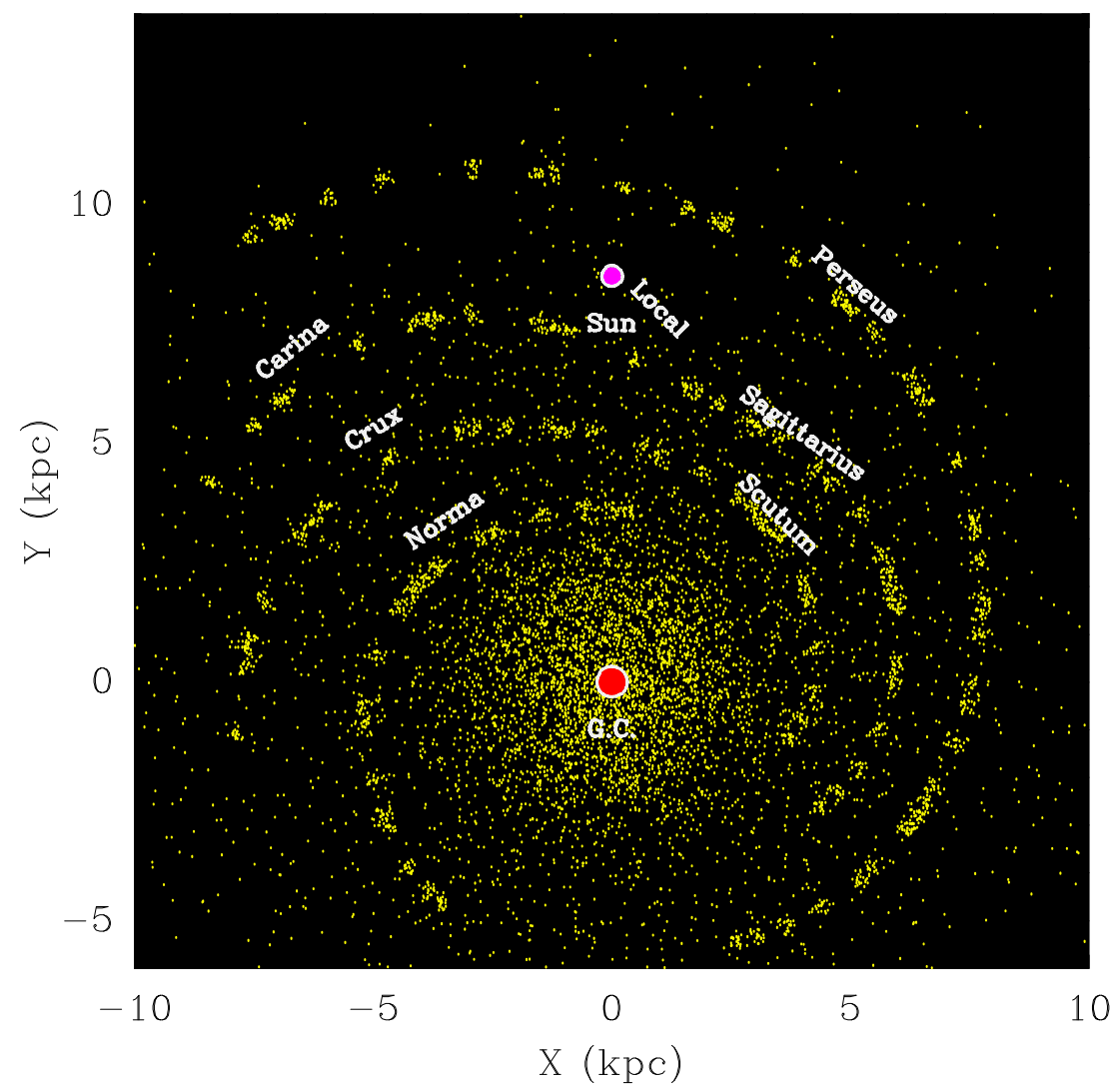

Figure 1: "Plan-view" model of the spiral structure of the Milky Way after Georgelin \& Georgelin (1976) and Taylor \& Cordes (1993). The main spiral arms are labeled. The red and purple dot indicate the position of the Galactic Center and the Sun, respectively.

of maser trigonometric parallaxes is the distance to the UCHII region $\mathrm{W} 3 \mathrm{OH}$ in the Perseus Arm, which has been indipendently measured using both methanol $(1.95 \pm 0.04 \mathrm{kpc}$; Xu et al. (2006)) and water $(2.04 \pm 0.07 \mathrm{kpc}$; Hachisuka et al. (2006)) masers, finding a very good agreement.

In addition to distances, the observations used to determine trigonometric parallaxes also yield excellent measurements of secular proper motions, with accuracies of $\approx 1 \mathrm{~km} \mathrm{~s}^{-1}$ (Xu et al. 2006). Combining radial velocity measurements with proper motions (and distances) yields full 3-dimensional velocities, relative to the motion of the Sun. Thus, maser VLBI observations can ultimately provide the full kinematics of massive star forming regions in the Milky Way, which will accurately define the rotation curve of the Milky Way and, in turn, its enclosed mass as a function of Galactocentric radius.

\section{Results}

Tables 1 and 2 list the sources whose parallax and proper motion have been so far measured by VLBA and VERA, respectively. The derived source distances are generally accurate to 5-10\%, and for three VLBA measurements (Orion, W3OH and S252) the accuracy is a few per cent. The accuracy in the proper motions generally correspond to uncertainties in maser spot velocities of a few kilometers per second. VLBA parallaxes listed in Table 1 have been determined by observing 
Table 1: VLBA parallax and proper motion measurements

\begin{tabular}{ccccc}
\hline \hline Source & $\begin{array}{c}\text { Parallax } \\
(\mathrm{mas})\end{array}$ & $\begin{array}{c}\mu_{\mathrm{x}} \\
\left(\mathrm{mas} \mathrm{y}^{-1}\right)\end{array}$ & $\begin{array}{c}\mu_{\mathrm{y}} \\
\left(\mathrm{mas} \mathrm{y}^{-1}\right)\end{array}$ & References \\
\hline Orion & $2.425 \pm 0.035$ & $3.3 \pm 1.0$ & $0.1 \pm 1.0$ & (Menten et al. 2007) \\
Cep A & $1.43 \pm 0.080$ & $0.5 \pm 1.1$ & $-3.7 \pm 0.2$ & (Moscadelli et al. 2008) \\
G 232.6+1.0 & $0.596 \pm 0.035$ & $-2.17 \pm 0.06$ & $2.09 \pm 0.46$ & (Reid et al. 2008) \\
W3OH & $0.512 \pm 0.010$ & $-1.20 \pm 0.2$ & $-0.15 \pm 0.2$ & (Xu et al. 2006) \\
S 252 & $0.476 \pm 0.006$ & $0.02 \pm 0.01$ & $-2.02 \pm 0.04$ & (Reid et al. 2008) \\
G 35.20-0.7 & $0.456 \pm 0.045$ & $-0.18 \pm 0.06$ & $-3.63 \pm 0.11$ & (Zhang et al. 2008) \\
G 59.7+0.1 & $0.463 \pm 0.020$ & $-1.65 \pm 0.03$ & $-5.12 \pm 0.08$ & (Xu et al. 2008) \\
NGC 7538 & $0.378 \pm 0.017$ & $-2.45 \pm 0.03$ & $-2.44 \pm 0.06$ & (Moscadelli et al. 2008) \\
G 35.20-1.7 & $0.306 \pm 0.045$ & $-0.71 \pm 0.05$ & $-3.61 \pm 0.17$ & (Zhang et al. 2008) \\
G 23.01-0.41 & $0.218 \pm 0.017$ & $-1.72 \pm 0.04$ & $-4.12 \pm 0.3$ & (Brunthaler et al. 2008) \\
W 51 IRS2 & $0.195 \pm 0.071$ & $-2.49 \pm 0.08$ & $-5.51 \pm 0.11$ & (Xu et al. 2008) \\
G 23.44-0.18 & $0.170 \pm 0.032$ & $-1.93 \pm 0.1$ & $-4.11 \pm 0.07$ & (Brunthaler et al. 2008)
\end{tabular}

Note.- Col. 1 gives the source name. Col. 2 the derived parallax. Cols. 3 and 4 the measured proper motion towards the East and North direction, respectively. Cols. 5 reports the bibliographic reference.

strong $12 \mathrm{GHz} \mathrm{CH}_{3} \mathrm{OH}$ masers associated with massive star-forming regions, with the exception of Orion toward which compact, non-thermal continuum sources were observed. The VERA results listed in Table 2 have been obtained by monitoring strong $22 \mathrm{GHz} \mathrm{H}_{2} \mathrm{O}$ masers.

Figure 2 illustrates the parallax fit for the source S 252 (Reid et al. 2008). Data presented are for two distinct maser spots whose motion across the sky is determined relative to the three background quasars: J0603+2159, J0607+2218 and J0608+2229. VLBA observed the $12 \mathrm{GHz}$ $\mathrm{CH}_{3} \mathrm{OH}$ masers in $\mathrm{S} 252$ at five epochs across one year, optimized to minimize correlation between parallax and proper motions. The global motion of a given maser spot can be modeled in terms of a linear motion (which derives from the sum of the apparent (solar + galactic) and peculiar motions) combined with a sinuisodal motion reflecting the earth revolution (i.e. the parallax signature).

\section{Discussion}

Combining the distances, LSR velocities and proper motions of the masers yields their locations in the Galaxy and their full space motions. Since internal motions of $12 \mathrm{GHz}$ methanol masers are fairly small, typically $\sim 3 \mathrm{~km} \mathrm{~s}^{-1}$ (Moscadelli et al. 2002), the maser motions should be close to that of their associated young stars. Given a model for the scale and rotation of the Milky Way, we can subtract the effects of Galactic rotation and the peculiar motion of the Sun from the space motions of the maser sources and estimate the peculiar motions of the maser star forming regions. We adopt the IAU values for the distance to the Galactic center $\left(R_{0}=8.5 \mathrm{kpc}\right)$ and the rotation 
Table 2: VERA parallax and proper motion measurements

\begin{tabular}{ccccc}
\hline \hline Source & $\begin{array}{c}\text { Parallax } \\
(\mathrm{mas})\end{array}$ & $\begin{array}{c}\mu_{\mathrm{x}} \\
\left(\mathrm{mas} \mathrm{y}^{-1}\right)\end{array}$ & $\begin{array}{c}\mu_{\mathrm{y}} \\
\left(\mathrm{mas} \mathrm{y}^{-1}\right)\end{array}$ & References \\
\hline & & & & \\
\hline O Oph & $5.6 \pm 1.0$ & $-20.6 \pm 0.7$ & $-32.4 \pm 2$ & (Imai et al. 2007) \\
NGC 1333 & $4.25 \pm 0.32$ & $17.9 \pm 0.9$ & $-7.9 \pm 1.4$ & (Hirota et al. 2008) \\
S Crt & $2.33 \pm 0.13$ & $-1.56 \pm 0.22$ & $-5.16 \pm 0.22$ & (Nakagawa et al. 2008) \\
Orion-KL & $2.29 \pm 0.1$ & $2.77 \pm 0.09$ & $-8.97 \pm 0.21$ & (Hirota et al. 2007) \\
VY CMa & $0.88 \pm 0.08$ & $-2.09 \pm 0.16$ & $1.02 \pm 0.61$ & (Choi et al. 2008) \\
NGC 281 & $0.355 \pm 0.030$ & $-2.63 \pm 0.05$ & $-1.86 \pm 0.08$ & (Sato et al. 2007) \\
S 269 & $0.189 \pm 0.010$ & $-0.422 \pm 0.010$ & $-0.121 \pm 0.042$ & (Honma et al. 2007) \\
& & & & \\
\hline
\end{tabular}

Note.- Col. 1 gives the source name. Col. 2 the derived parallax. Cols. 3 and 4 the measured proper motion towards the East and North direction, respectively. Cols. 5 reports the bibliographic reference.

speed of the Galaxy at this distance $\left(\Theta_{0}=220 \mathrm{~km} \mathrm{~s}^{-1}\right)$ and the Hipparcos measurements of the Solar Motion (Dehnen \& Binney 1998).

Fig. 3 shows the "plan-view" model of the spiral structure of the Milky Way (Georgelin \& Georgelin 1976; Taylor \& Cordes 1993) with overlaid positions and peculiar motions of the sources observed with VLBA (see Table 1) and the two most distant sources (S 269 and NGC 281) observed with VERA (see Table 2). Fig. 3 shows also the source WB89-437, for which a very accurate distance of $6.10 \pm 0.2 \mathrm{kpc}$ has been recently measured by Hachisuka et al. (2008), monitoring with the VLBA the associated $22 \mathrm{GHz} \mathrm{H}_{2} \mathrm{O}$ masers. The derived parallaxes allow us to confidently locate portions of the Perseus Arm (W3OH, NGC 7538 and S 252), the Local Spur (G 232.6+1.0, Orion, Cep A), the Carina-Sagittarius Arm (G 35.20-1.7, W 51 IRS2) and possibly the Crux-Scutum Arm (G 23.01-0.41). Comparison of trigonometric and kinematic distances indicate that in most cases kinematic distances are in excess, and this is particularly the case for the Perseus Arm sources affected by strong kinematic anomalies.

For most sources, the largest component of the derived peculiar motion is directed counter to Galactic rotation, that is these sources appear to rotate slower than the Galaxy spins (i.e. slower than circular rotation). The implications of these peculiar velocities for models of Galactic rotation and structure will be discussed in Reid et al. (in preparation).

The two most distant sources, S 269 and WB89-437, have a similar Galactocentric distance $\left(13.5 \mathrm{kpc}\right.$ ), and their positions support the presence of an Outer Arm extending from the $2^{\text {nd }}$ ( $l=$ $\left.135^{\circ}\right)$ to the $3^{r d}\left(l=196^{\circ}\right)$ Galactic quadrant. Looking at Fig. 3 one notes that in the direction of Galactic rotation the peculiar motion of both S 269 and WB89-437 is about zero. Since the peculiar motions in Fig. 3 are derived assuming a flat Galactic rotation curve, that indicates that at the Galactocentric radii of S 269 and WB89-437 $(13.5 \mathrm{kpc})$ the Galactic disk rotates at the same speed as at the Sun Galactocentric radius. This finding is a strong argument in favour of the presence of a large amount of dark matter in the outer region of the Galaxy, as discussed by Honma et al. (2007). 

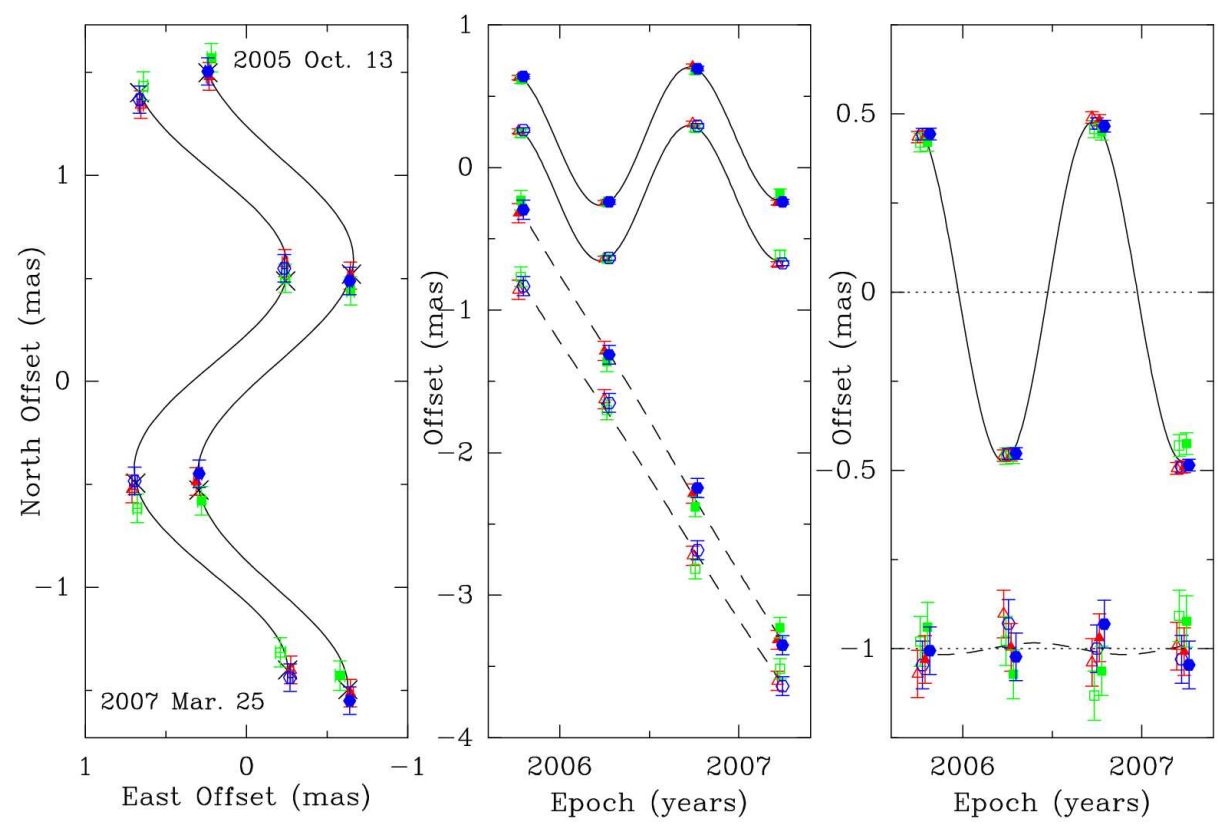

Figure 2: Parallax and proper motion data and fits for the source $S$ 252. Plotted are position measurements of two maser spots (open and solid symbols) relative to the three background sources: J0603+2159 (red triangles), J0607+2218 (green squares) and J0608+2229 (blue hexagons). Left Panel: Positions on the sky with first and last epochs labeled. Data for the two maser spots are offset horizontally for clarity. The expected positions from the parallax and proper motion fit are indicated (crosses). Middle Panel: East (solid lines) and North (dashed lines) position offsets and best fit parallax and proper motions fits versus time. Data for the two maser spots are offset vertical, the northward data have been offset from the eastward data, and small time shifts have been added to the data for clarity Right Panel: Same as the middle panel, except the with the best fit proper motions have been removed, allowing all data to be overlaid and the effects of only the parallax seen.

\section{Future Plans}

VERA plans to observe 70-80 maser sources every year, and in the next 12-15 years VERA will observe $\approx 1000$ maser sources to precisely locate them in the Galactic disk (Honma et al. 2008).

During 2008-2009, VLBA will observe another eight $12 \mathrm{GHz}$ maser sources selected such a way to better locate the Carina-Sagittarius Arm and the Crux-Scutum Arm. Additionally, VLBA will observe eight $22 \mathrm{GHz}$ water masers. Based on their kinematic distances, four of them are in the Outer Galaxy and the measurement of their distances and proper motions will constrain the rotation speed of the Milky Way at Galactocentric radii between 17 and $22 \mathrm{kpc}$. The remaining four water masers are in the Perseus Arm in the $1^{\text {st }}$ quadrant of the Milky Way, and will allow one to trace distant portions of this spiral arm.

Finally, it is worthwhile mentioning that the European VLBI Network is also measuring

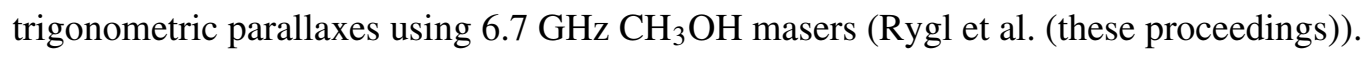




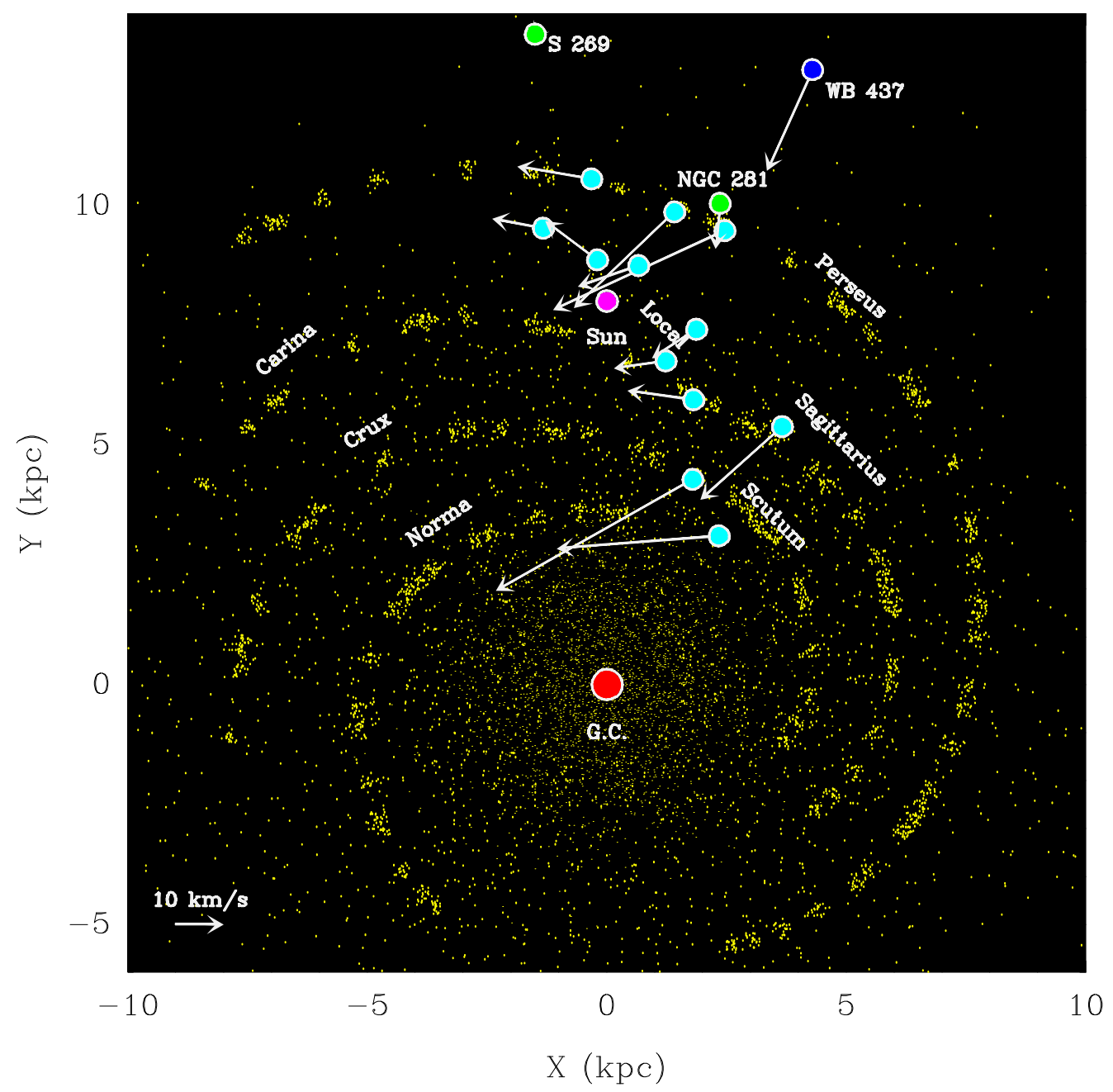

Figure 3: "Plain-view" model of the spiral structure of the Milky Way (Georgelin \& Georgelin 1976; Taylor \& Cordes 1993) with overlaid positions and peculiar motions of the sources observed with VLBA (light blue dots) (see Table 1) and the two most distant sources (S 269 and NGC 281) observed with VERA (green dots) (see Table 2). The source WB89-437 (blue dot), whose parallax and proper motion has been recently measured by Hachisuka et al. (2008) using VLBA observations of $22 \mathrm{GHz} \mathrm{H}_{2} \mathrm{O}$ masers, is also reported. The main spiral arms are labeled. The red and purple dot indicate the position of the Galactic Center and the Sun, respectively. Peculiar motions are calculated using a flat galactic rotation model with the IAU values of galactic constants $\left(R_{0}=8.5 \mathrm{kpc}, \Theta_{0}=220 \mathrm{~km} \mathrm{~s}^{-1}\right)$ and the Sun peculiar motion from Hipparcos data. The amplitude scale for the peculiar motions is given in the left bottom of the figure.

\section{References}

Bash, F. N., 1981, ApJ, 250, 551

Blitz, L., Fich, M., Kulkarni, S. 1983, Science, 220, 1233

Brunthaler, A., Reid, M. J., Menten, K. M., Zheng, X. W., Moscadelli, L. \& Xu, Y. 2008, ApJ accepted (arXiv:0811.0713). 
Burton, W. B. 1988, in "Galactic and Extragalactic Radio Astronomy (2 ${ }^{\text {nd }}$ edition), eds. K. I. Kellermann \& G. L Verschuur, ” Springer-Verlag, Berlin and New York, p. 295-358

Choi, Y. K., Hirota, T., Honma, M., \& Kobayashi, H. 2008, IAU Symposium 248, 192

Dame, T. M., Hartmann, Dap \& Thaddeus, P. 2001, ApJ, 547, 792

Dehnen, W. \& Binney, J. J. 1998, MNRAS, 298, 387

Georgelin, Y. M. \& Georgelin, Y. P. 1976, A\&A, 49, 57

Gerhard, O. 2002, SSRv, 100, 129.

Hachisuka, K. et al. 2006, ApJ 645, 337

Hachisuka, K., Brunthaler, A., Menten, K.M., Reid, J.M., Hagiwara, Y., Mochizuki, N. 2008, submitted to ApJ.

Hirota, T., et al. 2007, PASJ 59, 897

Hirota, M., et al. 2008, PASJ 60, 37

Honma, M. et al. 2007, PASJ 59, 889.

Honma, M. et al. 2008, IAU Symposium 248, 198

Imai, H. et al. 2007, PASJ 59, 1107

Kobayashi, H. et al. 2005, IAU Symposium 196, 496

Menten, K. M., Reid, M. J., Forbrich, J. and Brunthaler, A. 2007, A\&A 474, 515.

Moscadelli, L., Menten, K. M., Walmsley, C. M., \& Reid, M. J. 2002, ApJ, 564, 813

Moscadelli, L., Reid, M. J., Menten, K. M., Brunthaler, A., Zheng, X. W., \& Xu, Y. 2008, ApJ accepted (eprint arXiv:0811.0679).

Nakagawa, A., et al. 2008, IAU Symposium 248, 206

Reid, M. J., Menten, K. M., Brunthaler, A., Zheng, X. W., Moscadelli, L., \& Xu, Y. 2008, ApJ accepted (eprint arXiv:0811.0595)

Sato, M., et al. 2007, PASJ 59, 743

Taylor, J. H. \& Cordes, J. M. 1993, ApJ, 411, 674

Oort, J. H., Kerr, F. J., Westerhout, G. 1958, MNRAS, 118, 379

Xu, Y., Reid, M. J., Zheng, X. W. \& Menten, K. M. 2006, Science, 311, 54

Xu, Y., Reid, M. J., Menten, K. M., Brunthaler, A., Zheng, X. W., \& Moscadelli, L. 2008, ApJ accepted (eprint arXiv:0811.0701).

Zhang, B., Zheng, X. W., Reid, M. J., Menten, K. M., Xu, Y., Moscadelli, L. \& Brunthaler, A. 2008, ApJ accepted (eprint arXiv:0811.0704). 